\title{
X-RAY EMISSION FROM REVERSE-SHOCKED EJECTA IN SUPERNOVA REMNANTS
}

\author{
Denis F. Cioffit ${ }^{1 \dagger}$, and Christopher F. McKee ${ }^{2}$ \\ ${ }^{1}$ NASA/Goddard Space Flight Center, Greenbelt, Maryland, U.S.A. \\ ${ }^{2}$ University of California, Berkeley, California, U.S.A.
}

\begin{abstract}
A simple physical model of the dynamics of a young supernova remnant is used to derive a straightforward kinematical description of the reverse shock. With suitable approximations, formulae can then be developed to give the X-ray emission of the reverse-shocked ejecta. The results are found to agree favorably with observations of SN1006.
\end{abstract}

\section{Introduction}

Supernova remnants (SNRs) spend most of their lifetimes in stages where the mass of the swept interstellar material, $M_{s}$, dominates the mass of the ejecta, $M_{\mathrm{e} j}$, and where the radiation results from the cooling interior and from the actions of the main, forward shock. Before this occurs, however, radiation from reverseshocked ejecta in young SNRs provides the first evidence of the interaction between a remnant and the medium which surrounds it.

In Cioffi, McKee, and Bertschinger (1988), through a careful comparison with a hydrodynamical simulation, we developed accurate and smooth analytic kinematics of an expanding SNR from soon after the explosion until the remnant merges with the interstellar medium (ISM). In Cioffi and McKee (1988) we indicated how this improved understanding enables a better calculation of the evolving interior luminosity. In Cioffi and McKee (1989; hereafter CM) we shall present a comprehensive analytic treatment of the $\mathrm{X}$-ray luminosity from the interior and from the reverse-shocked ejecta. Here we show some results of our model of the reverse shock.

\section{The Dynamics}

Immediately after a supernova explosion, the pressure in the initially cold ejecta falls even further due to a rarefraction wave and the adiabatic expansion of the material. The strong pressure which lies behind the high-velocity forward shock drives a compression wave back into the ejecta, and that wave soon steepens into the reverse shock. Until the observations demonstrate the need for a more advanced approach, we rest our simple model (evolved from McKee [1974]) on the basic physics that govern the reverse shock interaction, and treat the problem analytically.

Our first assumption calls for uniform ejecta. Although both hydrodynamical simulations (e.g., Nomoto, Thielemann, and Yokoi 1984) and some supernova theory (e.g., Colgate and McKee 1969) predict an exponential fall-off in the density of

$\dagger$ NAS/NRC Resident Research Associate 
the ejecta, our apparent success, and that of Hamilton, Sarazin, and Szymkowiak (1986), in matching the X-ray observations of SN1006 suggest that only a small fraction ( $\lesssim 10 \%$ ) of the ejecta is found in this "tail" (e.g., Chevalier 1988), and most of the luminosity originates in the dense, uniform part.

We next assume that the pressure which drives the reverse shock remains a fixed fraction $\varphi$ of the pressure behind the forward shock,

$$
\rho_{e j} v_{r s}^{2}=\varphi \rho_{o} v_{s}^{2}
$$

where $v_{r s}$ is the velocity of the reverse shock, $v_{s}$ is the velocity of the forward shock, $\rho_{o}$ is the homogeneous mass density surrounding the supernova, and $\rho_{e j}$ is the uniform density of the unshocked ejecta. Hamilton and Sarazin's (1984) numerical simulation shows that the pressure ratio $\varphi$ varies smoothly with time, but for an average one might choose $\varphi \approx 0.2$. Setting $\varphi=0.3$, however, produces a better agreement (see the table below) with the dynamics found in Woosley's more sophisticated hydrodynamical model ("CDTG7") used by Hamilton and Fesen (1988).

\begin{tabular}{llll}
\multicolumn{4}{c}{ The Reverse Shock of SN1006 } \\
\multicolumn{1}{c}{ Parameter } & Unit & G7 & CM \\
\hline Shocked Fraction $F$ & & 0.80 & 0.95 \\
Blast-wave mass $M_{s}$ & $\mathrm{M}_{\odot}$ & 4.3 & 3.9 \\
Distance & $\mathrm{kpc}$ & 1.7 & 1.7 \\
Forward Shock Radius & $\mathrm{pc}$ & 7.4 & 7.3 \\
Blast-wave shock velocity & $\mathrm{km} \mathrm{s}$ & 4000 & 4195 \\
Reverse-shock velocity & $\mathrm{km} \mathrm{s}$ s $^{-1}$ & 4000 & 3939 \\
Density ratio $\rho_{e j} / \rho_{o}$ & \multicolumn{4}{c}{0.15} & 0.12 \\
\hline G7: "CDTG7" (Hamilton and Fesen $[1988])$ \\
CM: Cioffi and McKee (1989)
\end{tabular}

Our final assumption allows the outer ejecta radius to move with the velocity of the main shock, $v_{s}$. For an explosion of energy $E, v_{s}$ is assumed constant at $v_{e j}=\sqrt{2 E / M_{e j}}$ in the ejecta stage of evolution, and then smoothly slows into the Sedov-Taylor (ST) point-blast solution when $M_{s} / M_{e j}=2 / 3(\mathrm{CM})$.

With the definition $F=M_{r s} / M_{e j}$, where $M_{r s}$ is the ejecta mass which has been reverse-shocked, the above approximations eventually lead to

$$
\frac{M_{s}}{M_{e j}}=\frac{9}{4 \varphi}\left[1-(1-F)^{1 / 3}\right]^{2} .
$$

When $\varphi=0.3$, the ST stage begins at $F_{S T}=0.658(\mathrm{CM})$, so in this formalism SN1006 has entered the ST stage. ${ }^{1}$ The above table shows the excellent agreement between our analytic results and Hamilton and Fesen's (1988) hydrodynamical "CDTG7" computation when we use their best SN1006 model values of $M_{e j}=$ $1.32 \mathrm{M}_{\odot}, E=1.0 \times 10^{51} \mathrm{ergs}$, an ambient hydrogen number density of $0.069 \mathrm{~cm}^{-3}$, and an age of 980 years .

1 At $F=1, M_{s} / M_{e j}=7.5$ and the reverse shock hits the center, with reflected shocks presumably further complicating the interior. 


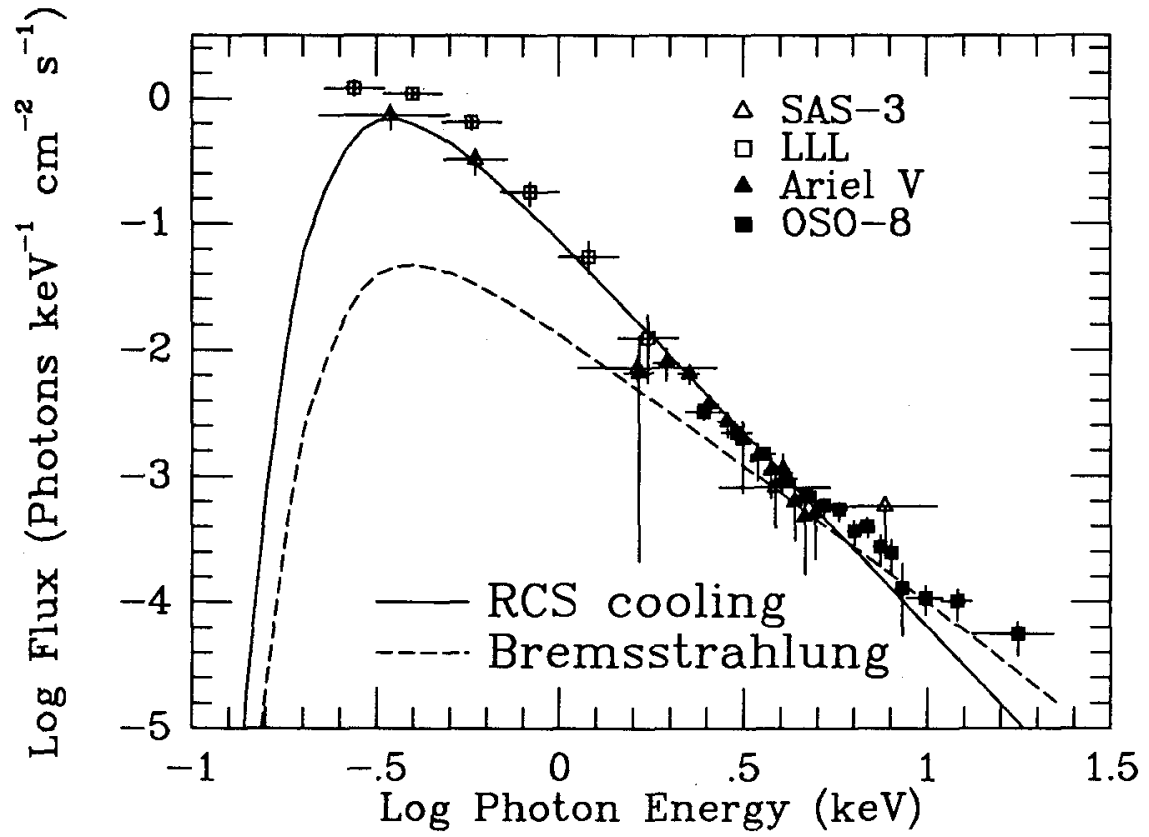

Observations of SN 1006 and the Reverse Shock Model

\section{The Luminosity}

The cooling of the ejecta produces a negligible decrease in the entropy of the gas during the dynamical timescales of interest, and thus we approximate an adiabatic shock for the luminosity calculation. We can write the total luminosity in terms of some appropriate cooling funcion $\Lambda(T)\left(\mathrm{ergs} \mathrm{cm}^{3} \mathrm{~s}^{-1}\right) \propto T^{m}$, defined so that the luminosity per unit volume is given by $n^{2} \Lambda$, where $n$ is the number density, and $m$ is either $-1 / 2$ (a fit to Raymond, Cox, and Smith [1976] cooling) or $+1 / 2$ (bremsstrahulung).

Although non-equilibrium ionization (NEI) typically increases the luminosity of the forward shock by a large factor (e.g., Shull 1982), its specific effect on the reverse-shocked material is less certain. The higher density of the ejecta may make NEI relatively unimportant (e.g., Itoh 1977), especially when compared to the effect of the metal-rich composition on the standard cooling curve, which we do attempt to account for (CM). (For SN1006 we assume pure carbon.)

For a parcel of hot gas at temperature $T$, the approximate luminosity above (photon) energy $\epsilon$ can be obtained from multiplying the total by $\exp \left[-\epsilon / k_{B} T(F)\right]$, where $k_{B}$ is Boltzmann's constant and $T(F)$ identifies the particular parcel of reverse-shocked material. We integrate through the shocked material in terms of the mass fraction $F$ and obtain

$$
L(t, \epsilon)=\int_{0}^{M_{r_{s}} / M_{e j}} d F n \Lambda[T(F)] \frac{M_{e j}}{m_{H}} \exp \left[-\epsilon / k_{B} T(F)\right],
$$

where $m_{H}$ is the mass of the hydrogen atom. To integrate the above, we need also assume a uniform pressure inside the reverse-shocked ejecta. For $\bar{P}_{e j}$ we use a 
fixed multiple of the immediate post-reverse-shock pressure, $\bar{P}_{e j}=f_{p}(3 / 4) \varphi \rho_{o} v_{s}^{2}$, where, from an average through Hamilton and Sarazin's (1984) similarity solution, we take $f_{p}=1.6$. We differentiate the integral with respect to $\epsilon$ to obtain a rough spectrum. The integrals are straightforward, but long, and we present both the complete expessions and useful approximations in $\mathrm{CM}$. For now we display the results through the particular example of SN1006.

The figure displays four sets of X-ray observations of SN1006, from Hamilton, Sarazin, and Szymkowiak (1986). Recent Tenma observations (not shown) suggest the possibility that these data are confused with emission from the Lupus region (see Koyama et al. 1987), which would steepen the spectrum above $1.5 \mathrm{keV}$. The solid line shows the results of the model using a fit to Raymond, Cox, and Smith (1976) cooling, and the dashed line shows our model's bremmstrahlung contribution.

\section{Summary}

The reverse-shock in young SNRs reheats the ejected material to X-ray temperatures. We have constructed a model for the kinematics of the reverse shock by using the simple dynamics expected from the supernova's interaction with a homogeneous ISM. With power-law cooling curves, we integrate through the reverseshocked ejecta to obtain the X-ray luminosity above any energy $\epsilon$ as function of time. Our agreement with observations of SN1006 illustrates the validity of this approach and suggests that it provides a useful tool for analyzing the physical conditions in young SNRs.

Acknowledgements. DFC thanks Andrew Hamilton for providing the SN1006 data in a convenient electronic form. CFM's research is supported in part by NSF grant AST 8615177.

\section{References.}

Chevalier, R. A. 1988, in Supernova Remnants and the Interstellar Medium, eds R. S. Roger and T. L. Landecker (Cambridge: Cambridge University Press), p. 31.

Cioffi, D. F., and McKee, C. F. 1988, in Supernova Remnants and the Interstellar Medium, eds R. S. Roger and T. L. Landecker (Cambridge: Cambridge University Press), p. 435.

Cioffi, D. F., and McKee, C. F. 1989, in preparation (CM).

Cioffi, D. F., McKee, C. F., and Bertschinger, E. 1988, Ap. J., 334, xxx.

Colgate, S. A., and McKee, C. 1969, Ap. J., 157, 623 .

Hamilton, A. J. S., and Fesen, R. A. 1988, Ap. J., 327, 164.

Hamilton, A. J. S., and Sarazin, C. L. 1984, Ap. J., 281, 682.

Hamilton, A. J. S., Sarazin, C. L., and Szymkowiak, A. E. 1986, Ap. J., 300, 698.

Itoh, H. 1977, Pub. Astr. Soc. Japan, 29, 813.

Koyama, K., Tsunemi, H., Becker, R. H., and Hughes, J. P. 1987, Pub. Astr. Soc. Japan, 39, 437.

McKee, C. F. 1974, Ap. J., 188, 335.

Nomoto, K, Thielemann, F-K., and Yokoi, K. 1984, Ap. J., 286, 644.

Raymond, J. C., Cox, D. P., and Smith, B. W. 1976, Ap. J., 204, 290.

Shull, J. M. 1982, Ap. J., 262, 308. 